\title{
Geovisualizing space and time in a science-art exhibit
}

\author{
Javier A. Arce-Nazario ${ }^{\mathrm{a}, \mathrm{b}} *$ \\ ${ }^{a}$ Department of Geography, The University of North Carolina at Chapel Hill, jarce@live.unc.edu \\ ${ }^{b}$ Institute of Interdisciplinary Studies, The University of Puerto Rico at Cayey \\ * Corresponding author
}

Keywords: space-time geovisualization, outreach, science-art exhibit, Puerto Rico

\begin{abstract}
:
The question of how to communicate with lay audiences about dynamic spatial processes is important in many disciplines. A diversity of paradigms for representing space and time have been developed in cartography, GIS science, and geovisualization, but these paradigms are unlikely to converge to a standard representation of spatiotemporal data (Goodchild 2013). Thus, finding the best visualization techniques to support the general public's understanding of spatiotemporal analysis requires some exploration. In the following, I discuss how this exploration produced the novel approaches to representing time and landscape dynamics in geo/visual/isla, which was a science-art exhibit about social and ecological changes in the landscape of Puerto Rico over the past century.

geo/visual/isla (Museo Casa Blanca, San Juan, 2017) was developed from static, large-format prints of aerial imagery of the Caribbean island nation of Puerto Rico, which were created by a collective of undergraduate students and a geographer at the University of Puerto Rico at Cayey. The data associated with times in the 1930s were derived from aerial photographs provided by the Puerto Rican Department of Transportation, and more recent data were derived from the United States Geological Survey and United States Army Corps of Engineers. The exhibit ultimately presented an 80-year history of changes in the natural and constructed landscape, during a period in which shifting global and local economies, migration, climate events and colonial policies were drivers of dramatic landscape transitions. The purpose of the exhibit was to capture the beauty and the dynamics of the landscape's history, while helping visitors to envision and discuss past landscape change and future land use in Puerto Rico.

The problem of geovisualizing time and change is an old one that has been extensively reviewed - for example, by Yuan (2016) - but when the audience is a general public, there are additional challenges. Most notably, the limited period of interaction that a lay person will have with the geospatial data in question means that the scheme for representing space and time together must be either simple or familiar to be successful. Many creators of geographic exhibits for lay audiences do utilize well-established geovisualization paradigms such as the space-time cube (Bogucka and Jahnke 2017), the time-animated series of maps (Harner, Knapp, and Davis-Witherow 2017), and the annotated timeline (Caine 2017). However, these techniques must be adapted for the intended audience: the authors in each case highlight the specific techniques they use to help viewers by reducing the information burden and interpretation ambiguity of the representations they choose.
\end{abstract}

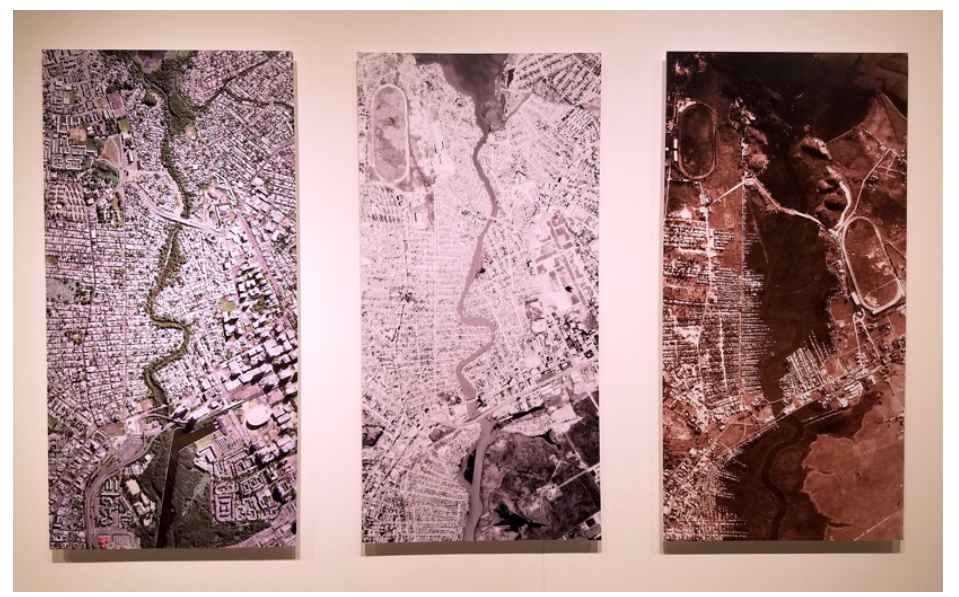

Figure 1. A triptych-style presentation of changes in a riverine landscape, in which the time coordinate (ca. 2010, 1950, 1930) is emphasized by a full-colour, black-and-white, or sepia rendering. 
Like these other public geographic exhibits, geo/visual/isla extensively used an early cartographic representation of time, which was chosen for its simplicity and familiarity. Several of the works in the exhibit were "time-slice snapshots," as described by Langran and Chrisman (1998). We took advantage of the rich vocabulary of the human experience of time to help viewers more easily navigate the temporal dimension of the data being displayed. For example, we encouraged viewers to associate neighboring time-slices by using the visual metaphor of the triptych, and used color schemes emphasizing the time coordinate (Figure 1). Spatial orientation between images was reinforced by choosing images with prominent, essentially consistent landscape features such as a coastline. The triptych format also reduced the temporal resolution to a manageable level, reducing the information burden noted above.

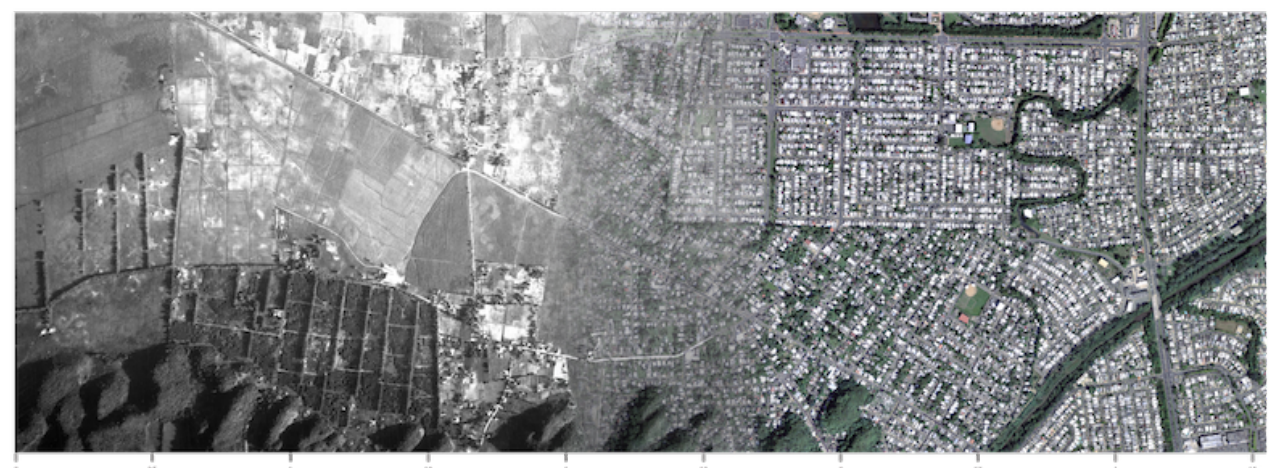

Figure 2. A blended image of landscape change in a relatively spatially homogenous landscape.

Perhaps the most important distinction between science-art exhibits and GIS representations or standalone geovisualizations is possibility to use the exhibit site as an additional dimension of experience. Harner, Knapp, and Davis-Witherow (2017) used this space for physical objects, and describe how their exhibit's interactive maps replace interpretation of these objects by curators. In geo/visual/isla, we chose the inverse relationship: the space itself provided orientation that helped viewers interpret the maps. This was achieved by two techniques: first, the viewers' path through the exhibit allowed them to learn the "vocabulary" of the space-time representation as they progressed. Timeslice snapshots gave way to more complex presentations where data with different space and time coordinates appeared in the same frame (Figure 2). By the end of the exhibit, viewers were easily able to read the spatial landscape enough to understand the story of change in these blended presentations. Second, the environment in different parts of the exhibit hall reinforced an understanding of timescales. Images in the exhibit depicting topological landscape features in the 1950s and 1960s were portrayed in red-blue anaglyph images and viewed with paper anaglyph glasses. In this corner of the exhibit, which was populated by other visitors in "retro" glasses and complemented by artworks referencing visual tropes of other dimensions and flashbacks, our intention was to make the actual ambiance provoke discussions of this particular period of Puerto Rico's past (Figure 3).
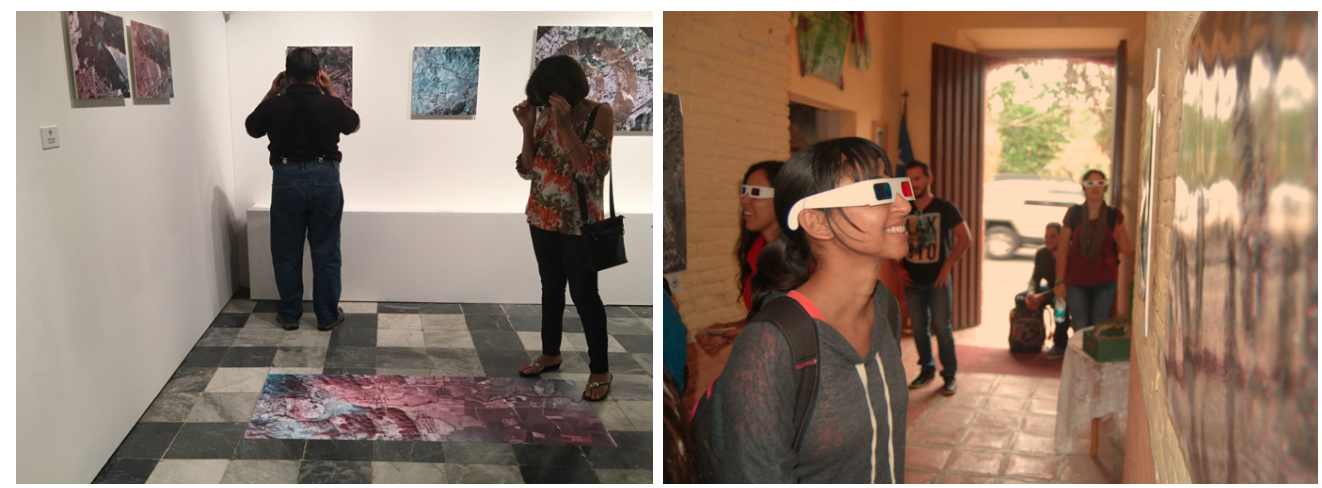

Figure 3. Left: The setting of the 1950s section of geo/visual/isla. The spiral overlay of data from two times, visible in the upper right, was inspired by classic film grammar. Right: Viewers using 3-d glasses like the ones in geo/visual/isla.

The techniques explored in geo/visual/isla made the dimensions of space and time equally easy to navigate for users, and our observation of visitors and their responses on surveys demonstrated that we successfully produced a conducive environment for substantive discussions of landscape change. The demonstrated effectiveness of the format is consistent with our visitor survey results from prior exhibitions (Arce-Nazario 2016). Our choices were specifically designed for a physical, artistic exhibit and a non-expert audience, but the training and cueing used to make geo/visual/isla work so well could also be adapted to other geovisualization presentations and tools. 